Marquette University

e-Publications@Marquette

School of Dentistry Faculty Research and

Publications

Dentistry, School of

$2-2-2018$

\title{
Collagenous Matrix Supported by A 3D-Printed Scaffold for Osteogenic Differentiation of Dental Pulp Cells
}

\author{
Farahnaz Fahimipour \\ Marquette University \\ Erfan Dashtimoghadam \\ Marquette University \\ Morteza Rasoulianboroujeni \\ Marquette University \\ Mostafa Yazdimamaghani \\ Oklahoma State University \\ Kimia Khoshroo \\ Marquette University \\ See next page for additional authors \\ Follow this and additional works at: https://epublications.marquette.edu/dentistry_fac \\ Part of the Dentistry Commons

\section{Recommended Citation} \\ Fahimipour, Farahnaz; Dashtimoghadam, Erfan; Rasoulianboroujeni, Morteza; Yazdimamaghani, Mostafa; \\ Khoshroo, Kimia; Tahriri, Mohammadreza; Yadegari, Amir; Gonzalez, Jose; Vashaee, Daryoosh; Lobner, \\ Douglas C.; Kashi, Tahereh S. Jafarzadeh; and Tayebi, Lobat, "Collagenous Matrix Supported by A 3D- \\ Printed Scaffold for Osteogenic Differentiation of Dental Pulp Cells" (2018). School of Dentistry Faculty \\ Research and Publications. 304. \\ https://epublications.marquette.edu/dentistry_fac/304
}




\section{Authors}

Farahnaz Fahimipour, Erfan Dashtimoghadam, Morteza Rasoulianboroujeni, Mostafa Yazdimamaghani, Kimia Khoshroo, Mohammadreza Tahriri, Amir Yadegari, Jose Gonzalez, Daryoosh Vashaee, Douglas C. Lobner, Tahereh S. Jafarzadeh Kashi, and Lobat Tayebi 
Marquette University

\section{e-Publications@Marquette}

\section{Dentistry Faculty Research and Publications/School of Dentistry}

This paper is NOT THE PUBLISHED VERSION; but the author's final, peer-reviewed manuscript. The published version may be accessed by following the link in th citation below.

Dental Materials, Vol. 34, No. 2 (February 2018): 209-220. DOI. This article is (C Elsevier and permission has been granted for this version to appear in e-Publications@Marquette. Elsevier does not grant permission for this article to be further copied/distributed or hosted elsewhere without the express permission from Elsevier.

\section{Collagenous Matrix Supported by a 3D- printed Scaffold for Osteogenic Differentiation of Dental Pulp Cells}

\section{Farahnaz Fahimipour}

Marquette University School of Dentistry, Milwaukee, WI

Dental Biomaterials Department, School of Dentistry, Tehran University of Medical Sciences, Tehran, Iran

Erfan Dashtimoghadam

Marquette University School of Dentistry, Milwaukee, WI

Morteza Rasoulianboroujeni

Marquette University School of Dentistry, Milwaukee, WI

Mostafa Yazdimamaghani

School of Chemical Engineering, Oklahoma State University, Stillwater, OK

Kimia Khoshroo

Marquette University School of Dentistry, Milwaukee, WI

Mohammadreza Tahriri

Marquette University School of Dentistry, Milwaukee, WI 


\section{Amir Yadegari}

Marquette University School of Dentistry, Milwaukee, WI

Jose A. Gonzalez

Marquette University School of Dentistry, Milwaukee, WI

Daryoosh Vashaee

Electrical and Computer Engineering Department, North Carolina State University, Raleigh, NC

Douglas C. Lobner

Department of Biomedical Sciences, Marquette University, Milwaukee, WI

\section{Tahereh S. Jafarzadeh Kashi}

Dental Biomaterials Department, School of Dentistry, Tehran University of Medical Sciences, Tehran, Iran

\section{Lobat Tayebi}

Marquette University School of Dentistry, Milwaukee, WI

Department of Engineering Science, University of Oxford, Oxford OX1 3PJ, UK

\section{Abstract \\ Objective}

A systematic characterization of hybrid scaffolds, fabricated based on combinatorial additive manufacturing technique and freeze-drying method, is presented as a new platform for osteoblastic differentiation of dental pulp cells (DPCs).

\section{Methods}

The scaffolds were consisted of a collagenous matrix embedded in a 3D-printed beta-tricalcium phosphate ( $\beta$ TCP) as the mineral phase. The developed construct design was intended to achieve mechanical robustness owing to 3D-printed $\beta$-TCP scaffold, and biologically active 3D cell culture matrix pertaining to the Collagen extracellular matrix. The $\beta$-TCP precursor formulations were investigated for their flow-ability at various temperatures, which optimized for fabrication of 3D printed scaffolds with interconnected porosity. The hybrid constructs were characterized by 3D laser scanning microscopy, X-ray diffraction, Fourier transform infrared spectroscopy, and compressive strength testing.

\section{Results}

The in vitro characterization of scaffolds revealed that the hybrid $\beta$-TCP/Collagen constructs offer superior DPCs proliferation and alkaline phosphatase (ALP) activity compared to the 3D-printed $\beta$-TCP scaffold over three weeks. Moreover, it was found that the incorporation of TCP into the Collagen matrix improves the ALP activity.

\section{Significance}

The presented results converge to suggest the developed 3D-printed $\beta$-TCP/Collagen hybrid constructs as a new platform for osteoblastic differentiation of DPCs for craniomaxillofacial bone regeneration.

\section{Graphical abstract}

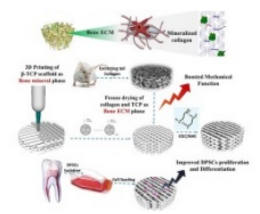




\section{Keywords}

Collagen, $\beta$-TCP, 3D-printing, Hybrid scaffolds, Dental pulp cells, Osteogenic differentiation

\section{Introduction}

Spontaneous regeneration capacity of bone tissue is only limited to small defects. Large bone defectsas consequence of trauma, osteoporotic fracture, tumors, and congenital deformity require surgical intervention [1]. Allografts and autografts are the main clinical strategies to fill bone cavities. However, autografts are restricted by site morbidity and approachability of the transplantable bone. Also, allografts increase the risk of immune-rejection reactions and infectious diseases transmission [2]. To address orthopedic challenges which results in reduced quality of life and patient discomfort, synthetic graft substitutes and implants have attracted tremendous interest in the last decade [3]. An ideal synthetic bone graft should satisfy a number of optimized properties such as biodegradability, osteoconductivity, biocompatibility, interconnected porosity, and adequate mechanical strength [4], [5], [6] in order to fulfill vascularization, nutrient delivery, cellular attachment, proliferation, differentiation, integration into surrounding tissue, to be ultimately replaced with the de novo bone tissue [7].

An ideal scaffold for bone tissue engineering application should mimic natural bone properties such as morphology, porosity, composition, and mechanical strength [8]. Naturally, bone is composed of hydroxyapatite $\left(\mathrm{Ca}_{10}\left(\mathrm{PO}_{4}\right)_{6}(\mathrm{OH})_{2}\right)$ in the context of organic matrix which is mainly comprised of Collagen type I [9]. Different bone regions possess specific microstructure and function. Proximal tibial trabecular bone has typically pore size of $1 \mathrm{~mm}$ in diameter, $50-90 \%$ porosity, $0.30 \pm 0.10 \mathrm{~g} / \mathrm{cm}^{3}$ apparent density, $5.3 \mathrm{MPa}$ ultimate strength, and $445 \mathrm{MPa}$ elastic modulus. In contrast, femoral cortical bone is compact with limited voids, 3-12\% porosity, $1.85 \pm 0.06 \mathrm{~g} / \mathrm{cm}^{3}$ apparent density, $193 \mathrm{MPa}$ ultimate strength, and $17 \mathrm{GPa}$ modulus of elasticity [9]. Bone is also a dynamic milieu, constantly repairing and remodelling its matrix by osteoblasts to create new mineralized bone matrix, osteoclasts disassembling and digesting the matrix, while the starshaped osteocytes maintaining the matrix [10]. Inspired by the hierarchical structure of the bone, the designed scaffolds for bone regeneration should structurally mimic the complexity of defected site and fulfill a 3D environment for initial cell attachment, consequent cell proliferation and tissue formation to ensure fixation and integration of scaffold with the host tissue [11], [12]. Generally, it has been suggested that fabrication of scaffolds with interconnected pores size over $300 \mu \mathrm{m}$ can facilitate cell attachment and migration, vascularization, mechanical interlocking, and tissue ingrowth [13].

There have been several fabrication techniques utilized to manufacture porous scaffold, including phase separation, salt leaching, freeze-drying, gas foaming, particle sintering, and solid free-form fabrication (SFF) techniques [14]. Conventional non-SFF approaches such as freeze-drying are limited in providing control on pore size, shape, interconnectivity, and construction of complex architectures [15], [16], [17], [18]. Furthermore, the low compressive moduli of these scaffolds hamper their application for the load bearing bone regeneration. Over the past few years, SFF techniques have gained extreme attention which the major sub-groups of this approach are selective laser sintering and 3D-printing[19], [20]. Additive layer manufacturing (ALM) or 3Dprinting techniques offer the advantage of creating desired levels of complexity in custom made implants according to the medical requirements [21]. In fact, 3D-printing method could be utilized in personalized medication level by obtaining individual patient computed tomography scan or magnetic resonance images [22], [23]. Indeed, 3D-printing is known as a reproducible and consistent technique. The major advantages and disadvantages of different classification of ALM techniques for bone tissue engineering applications have been thoroughly summarized elsewhere [24]. 3D-printing technique enables fabrication of well-defined complex architectures both in micro and macro scales with improved mechanical properties, tuneable for different application such as soft or hard tissues [25]. In spite of several advantages, 3D-printing 
suffers from the lack of ability to mimic nano-fibrous structure of extracellular matrix (ECM) which mainly supports cell adhesion, proliferation, communication, and differentiation. The advantages and disadvantages of conventional non-ALM approaches and 3D-printing, have motivated researches to exploit combinatorial fabrication techniques in order to mimic the nature of bone tissue [26].

The present work aims to employ 3D-printing technique and freeze-drying method to design optimal platforms for harnessing osteogenic capacity of DPCs. To achieve this goal, hybrid constructs consisted of 3D-printed $\beta$ tricalcium phosphates ( $\beta$-TCP) scaffolds filled with freeze-dried Collagen matrix were fabricated. The $\beta$-TCP was selected due to its chemical likeness to the bone mineral, which is commonly used in bone replacement applications. The in-depth physicomechanical and biological features of the developed $\beta$-TCP/Collagen hybrid constructs were investigated toward craniomaxillofacial bone regeneration.

\section{Materials and methods}

\subsection{Ink preparation and optimization}

The paste formulations were composed of water, beta-tricalcium phosphate powder ( $\beta$-TCP, Sigma), sodium tripolyphospahte (TPP, Alfa Aesar), and carboxymethylcellulose (CMC, Alfa Aesar). The TCP-based formulations were composed of $15 \mathrm{~g}$ TCP powder, $0.5 \mathrm{~g}$ TPP, $75 \mathrm{mg} \mathrm{CMC}$, and 5.25, 5.75, and $7.75 \mathrm{ml}$ water, which denoted as formulation 1, 2, and 3. The ink formulations were characterized to optimize their flow-ability for the 3Dprinting process. Rheological measurements were performed by a rheometer (Kinexus, Malvern) using a coneplate geometry. Shear stress and viscosity were measured altering formulations temperature from 17 to $29^{\circ} \mathrm{C}$, and shear rate of $0-1001 / \mathrm{s}$.

\subsection{Fabrication of $\beta$-TCP scaffolds using 3D-printing technique}

3D-bioplotting system (Envisiontec, Germany) was utilized to fabricate scaffolds with desired geometry, size, and structure. A CAD/CAM software was used to design disk block models of scaffold. The 3D-printing was performed on paste formulations at $5 \mathrm{~mm} / \mathrm{s}$ dispensing speed, optimal pressure of $1.5 \mathrm{bar}$, and a plotting needle (Nordson, USA) owning an inner diameter of 400 um to obtain $0.6-0.8 \mathrm{~mm}$ distanced strands with $90^{\circ}$ shifted between layers. Disc of $15 \mathrm{~mm}$ diameter and $2 \mathrm{~mm}$ thickness were prepared in a layer-by-layer deposition of strands from the printer head. The fabricated scaffolds were air dried overnight, and sintered in controlled high temperature muffle furnace (MTI, USA). A sequential temperature profile was used for sintering the 3D-printed scaffolds as follows: heating up uniformly to $600{ }^{\circ} \mathrm{C}$ at $3^{\circ} \mathrm{C} / \mathrm{min}$, incubation for $1 \mathrm{~h}$ at $600^{\circ} \mathrm{C}$, heating up from 600 to $1100^{\circ} \mathrm{C}$ at $5^{\circ} \mathrm{C} / \mathrm{min}$, and incubation at $1100^{\circ} \mathrm{C}$ for $4 \mathrm{~h}$.

\subsection{X-ray diffraction analysis}

X-ray diffraction (XRD) was carried out to characterize the TCP powder and scaffold. Sintered scaffolds were ground using an agate mortar and pestle. The powder was then sieved to $150 \mu \mathrm{m}$ particles.

\subsection{Isolation and characterization of rat tail Collagen type I}

Collagen type I was extracted from Wistar rat tail based on a protocol described elsewhere [27]. In brief, bone and tendons were exposed and removed by folding the tails. After washing with PBS, tendons were dissolved in $0.1 \mathrm{M}$ autoclaved acetic acid at $4{ }^{\circ} \mathrm{C}$ for 7 days. Collagen foam was then obtained by freeze-drying the solution. A stock solution with $5 \mathrm{mg} / \mathrm{ml}$ concentration was prepared by re-dissolving the Collagen foam in $0.1 \mathrm{M}$ acetic acid aqueous solution, and stored at $4{ }^{\circ} \mathrm{C}$ for further usage.

\subsection{Differential scanning calorimetry}

Differential scanning calorimetry technique (DSC, NETZCH) was employed with scan rate of $10^{\circ} \mathrm{C} / \mathrm{min}$ to identify the extracted Collagen denaturation temperature. The freeze-dried extracted Collagen samples were 
characterized through two methods, one was direct heating up from the room temperature, and the other was heating up from room temperature to $130{ }^{\circ} \mathrm{C}$, isothermal incubation for $1 \mathrm{~h}$, and heating up to $250^{\circ} \mathrm{C}$ to eliminate the effect of residual hydration.

\subsection{Fabrication of $\beta$-TCP/Col hybrid constructs}

The prepared Collagen solution $\left(5 \mathrm{mg} \mathrm{ml}^{-1}\right)$ described above in pure state or mixed with $\beta$-TCP powder ( $50 \mathrm{wt} \%$ of the dry weight of Collagen) was filled into pores of the 3D-printed $\beta$-TCP scaffolds, and then frozen by 10 min immersion in liquid nitrogen. Subsequently, the frozen mixture was lyophilized to produce $\beta$-TCP/Collagen hybrid construct. The Collagen-based matrices formed inside the scaffold pores were further crosslinked using 1(3-dimethylaminopropyl)-3-ethylcarbodiimidehydrochloride (EDC, TCI Chemicals) and hydroxysuccinimide (NHS, Alfa Aesar) to increase their stability. The constructs were denoted as $\beta-T C P / C o l$ and $\beta-T C P / C o l-T C P$.

\subsection{Morphological characterization of $\beta$-TCP scaffolds and $\beta-\mathrm{TCP} / \mathrm{Col}$ hybrid constructs} The microstructure and morphology of the $\beta$-TCP scaffolds and $\beta$-TCP/Col hybrid constructs were assessed by a 3D laser scanning digital microscope (Olympus LEXT OLS 4000, Japan). At least 3 different sites of 3D-printed scaffolds and hybrid constructs were randomly selected to measure the dimensions of the pore size, thickness of strands, distance between strands, and other microstructural features using the image software (LEXT OLS 4000).

\subsection{Fourier transform infrared spectroscopy}

Fourier-transform infrared (FT-IR) spectroscopy (Nicolet iS5 Thermo Scientific ${ }^{\mathrm{TM}}$ USA, OMNIC'M ) was performed to identify the functional vibrations groups in the fabricated constructs over the range of 4000 $600 \mathrm{~cm}^{-1}$ wavenumber at a resolution of $4 \mathrm{~cm}^{-1}$.

\subsection{Porosity and mechanical properties}

Porosity was measured using solvent displacement method as previously reported [28]. Briefly, scaffolds were weighed in dry state and then immersed in $10 \mathrm{ml}$ ethanol. The specimens containing ethanol were weighed and porosity of scaffolds was calculated using the following equation:Porosity $=(W w-W d) \times 100 \% \rho \times \pi R 2 T w h e r e$ $W_{w}$ and $W_{d}$ are the weight of scaffold in wet and dry state, $\rho$ is the solvent density, and $R, T$ are the radius and thickness of scaffolds, respectively.

The compressive modulus and strength of the Collagen-based matrices and $\beta$-TCP/Col constructs were measured utilizing a mechanical testing machine (Shimadzu, Japan) with cross-head speed of $1.0 \mathrm{~mm} \mathrm{~min}^{-1}$ and a $5 \mathrm{kN}$ load cell. The cylindrical samples $(n=5)$ were compressed until they crushed. Furthermore, the compressive modulus of the 3D-printed $\beta$-TCP scaffolds after immersion in PBS was measure over 4 weeks.

\subsection{Isolation and characterization of dental pulp cells}

Dental pulp stem cells from the pulp tissue were isolated to study cell attachment, proliferation, and differentiation on the fabricated scaffolds and constructs. According to a protocol reported elsewhere, normal impacted third molars were washed several times with PBS containing $100 \mathrm{mg} / \mathrm{ml}$ streptomycin and $100 \mathrm{U} / \mathrm{ml}$ penicillin. Pulp tissues were removed, transferred to sterile dish and then cut into small pieces. An enzyme solution consisted of $4 \mathrm{mg} / \mathrm{ml}$ dispase (Sigma), and $3 \mathrm{mg} / \mathrm{ml}$ type I collagenase (Sigma) in Hank's Balanced Salt Solution (HBSS, Sigma) were used to solubilize the pulp tissue for $1 \mathrm{~h}$ at $37^{\circ} \mathrm{C}$ with a regular agitation. The cell suspension was added to $5 \mathrm{ml}$ of alpha-minimal essential medium ( $\alpha \mathrm{MEM}$, Sigma) supplemented with 15\% FBS (Sigma), and centrifuged at $300 \mathrm{~g}$ for $5 \mathrm{~min}$. Afterward, the products were centrifuged and pellet was suspended in fresh aMEM supplemented with $15 \%$ FBS. The isolated dental pulp cells (DPCS) were then cultured into tissue culture polystyrene (TCPS) and incubated at $5 \% \mathrm{CO}_{2}$ and $37^{\circ} \mathrm{C}$ for further experiments. 
Alizarin red assay was performed to confirm osteogenic capability of DPCs. The isolated DPCs at passage three were cultured on TCPS for 3 weeks in osteogenic medium, consisting of $\alpha$ MEM supplemented with 10\% FBS, $50 \mathrm{\mu g} / \mathrm{ml}$ ascorbic acid, $10 \mathrm{mM} \beta$-glycerophosphate and $10 \mathrm{nM}$ dexamethasone and then fixed, washed and stained with alizarin red staining solution. In brief, $40 \mathrm{mM}$ Alizarin Red S (Alfa Aesar) solution was prepared and the $\mathrm{pH}$ adjusted to 4.2 with $10 \%$ ammonium hydroxide. Cells were fixed with $1 \mathrm{ml}$ formaldehyde for $10 \mathrm{~min}$ and then stained with $1 \mathrm{ml}$ of the prepared solution for $30 \mathrm{~min}$.

The DPCs were also characterized for their cell-surface markers expression by fluorescence activated cell sorting (FACS) technique (Attune ${ }^{\circ}$ Acoustic Focusing Cytometer, Thermo Fisher Scientific). A single cell suspension was prepared using cell strainer $\left(70 \mu \mathrm{m}, \mathrm{VWR}^{\oplus}\right.$ Cell Strainers) and aliquoted at $5 \times 10^{5}$ cells into the FACS tubes. Subsequently, the cells were resuspended in 3\% BSA blocking buffer and incubated for 30 min in darkness with isothiocyanate-conjugated anti-CD90 (CD90-FITC), phycoerythrin-conjugated anti-CD34 (CD34PE), FITC-conjugated anti-CD105 (CD105-APC), FITC-conjugated anti-CD44 (CD44-FITC), and PE-conjugated antiCD45 (CD45- PE). All antibodies were purchased from BD Biosciences. Finally, cells were washed twice with PBS and resuspended in $500 \mu \mathrm{l}$ PBS containing $1 \%$ FBS. The obtained results were analysed by Attune flow cytometer software.

\subsection{Cell adhesion}

At the first step, scaffolds were disinfected by three times immersion in $70 \%$ ethanol followed by rinsing with PBS for 15 min cycles and air dried. $5 \times 10^{5}$ cells were then seeded on the scaffolds and immersed in growth medium which was changed every other day. The attachment and morphology of cells on the scaffolds was examined by scanning electron microscopy (SEM). To do this, scaffolds were removed from the culture medium, washed in PBS, and fixed with $4 \%$ of glutaraldehyde. The seeded constructs were then dehydrated in gradient ethanol concentrations of 50, 70, 80, 90 and $100 \%$ to conserve their intact morphology. Samples were coated with gold by means of a GSL-1100X-SPC12 Compact Plasma Sputtering Coater instrument, and scanned for their microstructure using scanning electron microscope (SEM, JEOL JSM-6510LV).

For immunocytochemical staining, following fixation in formalin the cells were permeabilized and the Factin Staining Kit-Cytopainter (abcam 112125) was used. The actin cytoskeleton of the cells attached to the scaffold stained with green fluorescent phalloidin conjugate for $1 \mathrm{~h}$, with the nuclei counterstained with $\underline{4,}, 6$ diamidino-2-phenylindole dihydrochloride (DAPI). Cell distribution and morphology were evaluated under a fluorescence microscope (Evos Flueroscent).

\subsection{Cell proliferation}

PrestoBlue (PB) cell vitality assay (Invitrogen, USA) was carried out according to the manufacturer's instructions at different time intervals of 7, 14 and 21 days after cell culture. The scaffolds were disinfected using $70 \%$ ethanol as described above. Afterward, $1 \times 10^{6}$ cells were seeded onto the scaffolds which were placed in a nonadherent 12 well-plate and then submerged in culture medium. The medium was changed every other day. After the incubation period, $10 \%$ Presto blue was added to the scaffold-call complexes. The fluorescence measurements (Ex: $560 \mathrm{~nm}$ and Em: $590 \mathrm{~nm}$ ) were determined by a spectrophotometric plate reader (Synergy HTX, BioTEK). In order to record the difference between experimental groups. Triplicate samples were analyzed for this experiment.

\subsection{Cell differentiation}

Alkaline phosphatase (ALP) activity of DPCs cultured on scaffolds was implemented utilizing an ALP assay kit (Abcam, USA). The samples were incubated in osteogenic medium and tested after 7, 14 and 21 days to investigate osteogenic differentiation of DPCs. The $50 \mu$ l of lysate was reacted with $150 \mu$ l of $p$-nitrophenyl phosphate (p-NPP) for $30 \mathrm{~min}$ at $37^{\circ} \mathrm{C}$. The reaction was terminated with stop solution and the absorbance of $p$ - 
nitrophenol was measured at $405 \mathrm{~nm}$ employing a microplate reader (Synergy HTX, BioTEK) to indicate the ALP quantity.

Cell lysates were analyzed for protein content using a micro-BCA Assay kit (Pierce), and ALP activity was normalized to the total protein content, which was measured using Pierce BCA protein assay kit. Alkaline phosphatase activity is expressed in arbitrary units. The Results represent the mean values of three individual experiments and each in quadruplicate.

\subsection{Statistical analysis}

All data were expressed as mean \pm standard deviation (Mean \pm SD). The significance difference was calculated by one-way ANOVA complemented by Tukey's multiple comparisons test. $P$-values $<0.05$ were considered to be statistically significant.

\section{Results}

In order to obtain a $\beta$-TCP-based paste formulation with appropriate flowability to be used in the 3Dprinting process, their rheological features including shear stress and shear viscosity were studied at various temperatures. Shear stress as a function of shear rate for the paste formulations is shown in Fig. $1 \mathrm{~A}-\mathrm{C}$, which indicates a Bingham pseudoplastic behavior for the paste formulations. All formulations were found to show a yield stress, which is required for the 3D-printing process. As can be seen in Fig. 1D-F, the highly $\beta$-TCP filled paste inks exhibits shear-thinning behavior as shear viscosity decreases with increasing shear rate. It was found that with increasing water content in paste formulations shear viscosity decreases indicating the augmentation of flowability of the paste formulations. Based on the obtained results, the formulation 2 was selected for 3Dprinting process, which was found to fulfill reliable dispensing at pressure of 1.5 bar while maintaining the strand shape. In other words, formulation 2 was figured out to have the minimum amount of water to provide aforementioned paste features for the 3D-printing process. It is worthy of note that the formulation 2 showed the lowest dependence on temperature alteration, which helps additive layer manufacturing of the $\beta$-TCP scaffolds in a reproducible manner.
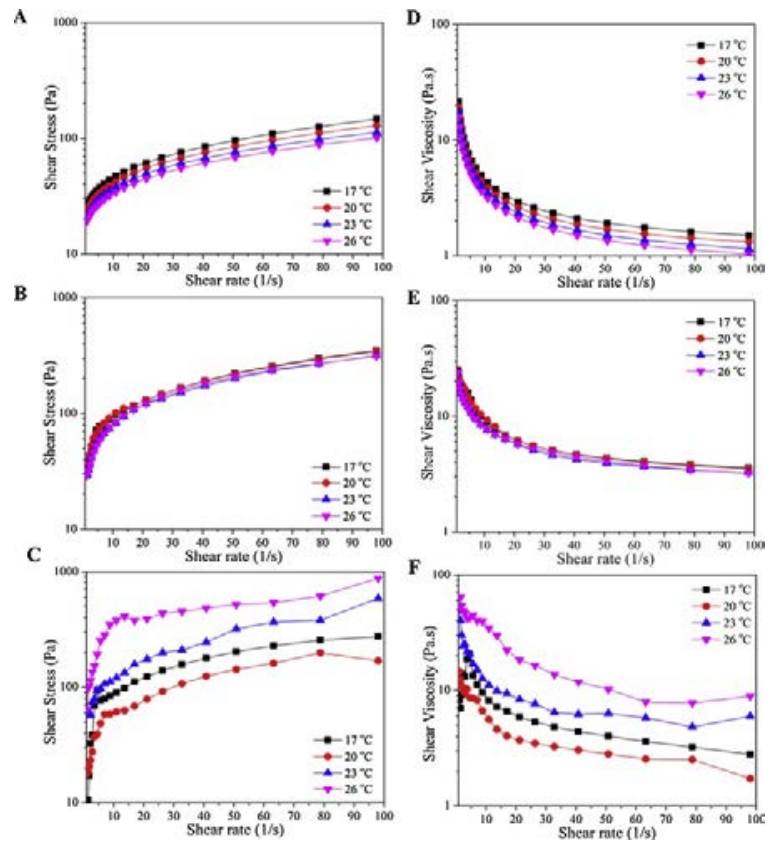

Fig. 1. (A-C) Shear stress as a function of shear rate at various temeartures for $\beta$-TCP-based paste formulations (A: formulation 1, B: formulation 2, and C: formulation 3). (D-F) Dependance of shear viscosity on shear rate at 
different temeartures for the $\beta$-TCP-based paste formulations (D: formulation 1, E: formulation 2, and F: formulation 3).

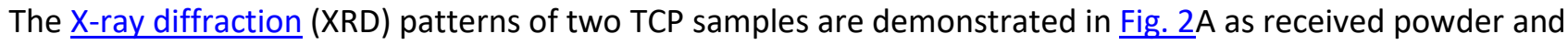
ground sintered 3D-printed TCP scaffolds. As can be seen, both samples show the characteristic peaks of the primarily $\beta$-TCP phase [29].
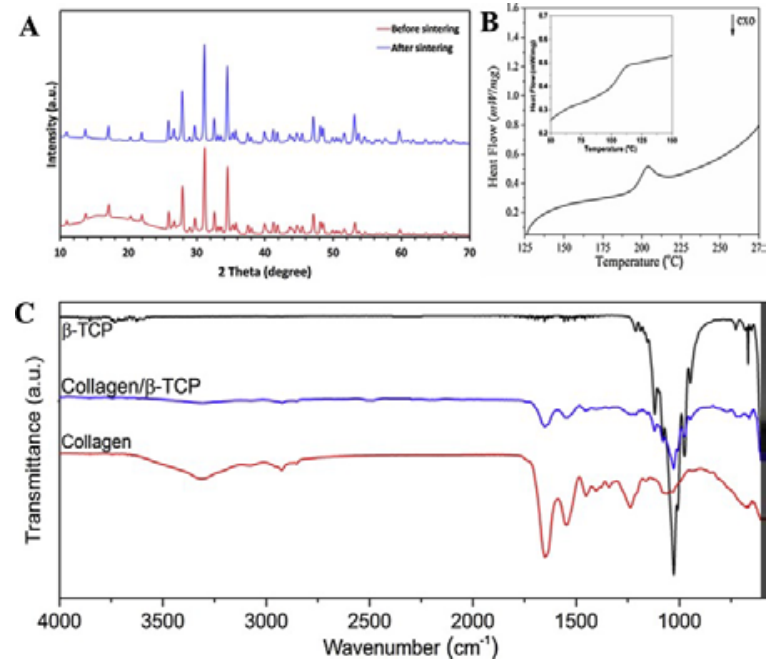

Fig. 2. (A) The X-ray diffraction patterns of TCP samples, as received $\beta$-TCP powder in comparison with the ground sintered 3D-printed TCP scaffolds (The inset shows a schematic representation of 3D-printed $\beta$-TCPbased scaffolds). (B) Differential scanning calorimetry (DSC) thermogram of fully dehydrated freeze-dried Collagen matrix (The inset displays the corresponding DSC thermogram for the freeze-dried Collagen matrix). (C) Fourier transform infrared spectra of $\beta$-TCP, Collagen, and Collagen-TCP composite samples.

The isolated Collagen (Col) was characterized through differential scanning calorimetry (DSC) at fully dried and partially hydrated states. The DSC thermograms are shown in Fig. $2 \mathrm{~B}$, where the distinctive peaks are attributed to the denaturation of the Collagen chains. In order to eliminate the plasticizing effect of residual water on denaturation, the freeze-dried Collagen sample went through the following heating steps: heating up from room temperature to $130{ }^{\circ} \mathrm{C}$, isothermal incubation for $1 \mathrm{~h}$, and finally heating up to $250^{\circ} \mathrm{C}$. As can be seen in Fig. $2 \mathrm{~B}$, the onset and peak temperatures were measured to be about 193.7 and $203.9^{\circ} \mathrm{C}$, respectively. In order to evaluate the Collagen matrix comprising structural residual water, the freeze-dried samples were heated up without isothermal step. In this way, the onset and peak temperatures were obtained as 102.5 and $113.1{ }^{\circ} \mathrm{C}$, respectively (see the inset shown in Fig. 2B).

Fig. $2 \mathrm{C}$ shows the FT-IR spectra of Collagen, $\beta$-TCP, and Collagen-TCP composite matrix. As seen, the $\mathrm{C}=\mathrm{O}$ stretching of carboxamide (amid I) functional groups along the polypeptide backbone observed at $1653 \mathrm{~cm}^{-1}$. The distinctive peaks observed at 1546 and $1234 \mathrm{~cm}^{-1}$ are related to $\mathrm{N} \_\mathrm{H}$ bending vibrations and $\mathrm{C} \_\mathrm{H}$ stretching of amide II and III bands, respectively. In addition, stretching of $\mathrm{N}-\mathrm{H}$ groups coupled with hydrogen bonding (amid A) and amid B bonding ( $\mathrm{C}$ _ $\mathrm{H}$ stretching) at broad band ranging from 3200 to $3600 \mathrm{~cm}^{-1}$ is ascribed to the Collagen characteristic peak [30]. The spectrum of $\beta$-TCP exhibited the characteristic spectral bands at 1119, 1079, 1027, 976 and $947 \mathrm{~cm}^{-1}$ attributed to vibrational mode of u3PO43- [31]. As can be seen, both characteristics peaks of the Collagen and $\beta$-TCP are observed in the FT-IR spectrum of Collagen-TCP composite sample.

The schematic representation of the developed combinatorial approach to fabricate hybrid constructs consisted of freeze-dried Collagen matrix supported by 3D-printed $\beta$-TCP scaffold are shown in Fig. 3A. The macro- to microscale structural features of 3D-printed $\beta$-TCP scaffold as well as 3D-printed $\beta$-TCP/Col hybrid constructs are 
shown in Fig. 3B-G, respectively. As seen in Fig. 3B-D, the optimized paste formulation 2 has allowed to obtain precise printing of $\beta$-TCP scaffolds, acquiring mechanical function of the hybrid constructs. As observed in Fig. $\underline{3} E-G$, the hybrid construct is mainly composed of a freeze-dried Collagen matrix with interconnected porosity inside the pores of 3D-scaffold to provide a platform for cell attachment and growth.

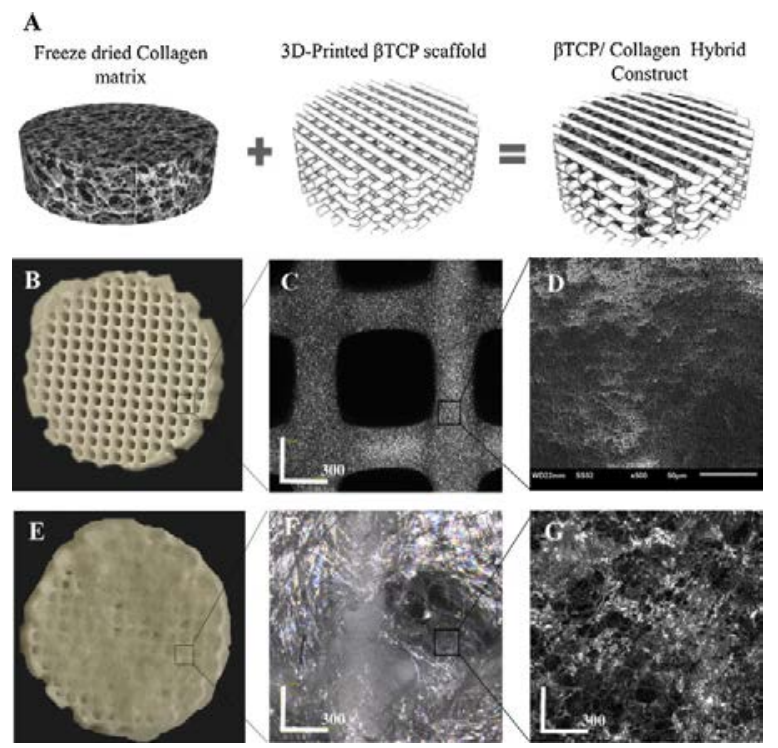

Fig. 3. (A) Schematic representation of the combinatorial approach developed to fabricate hybrid constructs consisted of freeze-dried collagen matrix supported by 3D-printed $\beta$-TCP scaffold. (B-D) Macro- to microscale demonstration of structural features of 3D-printed $\beta$-TCP scaffold. (E-G) Macro- to microscale demonstration of structural features of 3D-printed $\beta-\mathrm{TCP} /$ Col hybrid constructs.

The 3D laser scanning micrographs of the 3D-printed $\beta$-TCP scaffold is shown in Fig. 4A. As can be seen, the pore size of the scaffolds was found to be in the range of $426 \pm 17 \mu \mathrm{m}$. The compressive modulus of Collagen and Collagen-TCP matrices were compared with $\beta$-TCP/Col and $\beta-T C P / C o l-T C P$ hybrid constructs in Fig. 4B. As evident in Fig. 4C, biodegradation of scaffolds have resulted in decreased compressive modulus over time, which was found to become more pronounced after 2 and 4 weeks. However, it should be noted that after four weeks of immersion, scaffold still maintain over $30 \%$ of their initial compressive modulus providing host tissue enough time for ingrowth into the constructs.
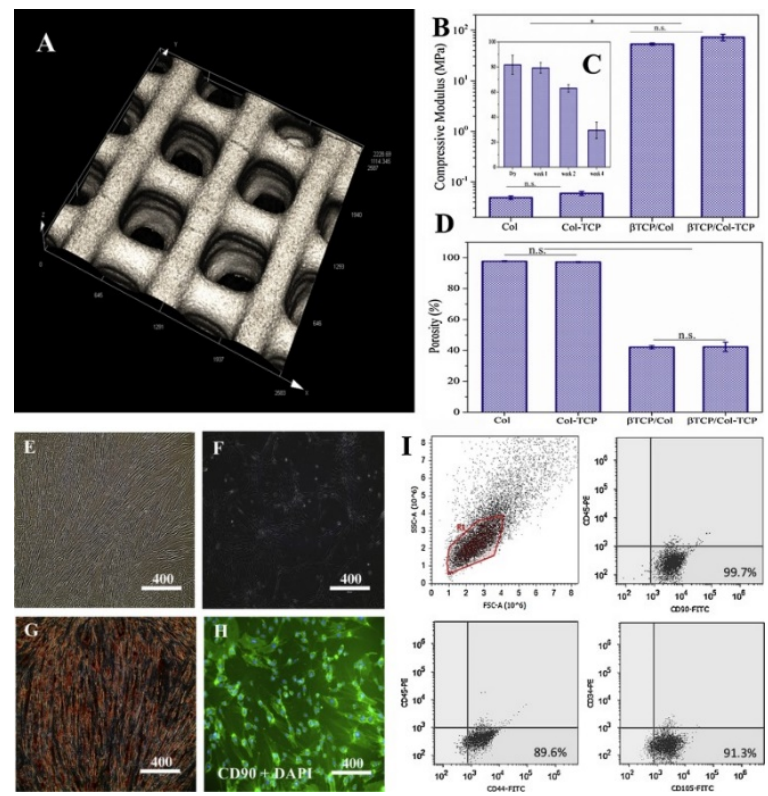
Fig. 4. (A) 3D laser scanning micrographs of the 3D-printed $\beta$-TCP scaffold (The scale bar is $500 \mu \mathrm{m}$ ). (B) Compressive modulus of Collagen and Collagen- $\beta$-TCP matrices compared with $\beta-\mathrm{TCP} / \mathrm{Col}$ and $\beta-\mathrm{TCP} / \mathrm{Col}-\mathrm{TCP}$ hybrid constructs. (C) Compressive modulus of 3D-printed $\beta$-TCP scaffolds in dry state and after 1,2 , and 4 weeks immersion in phosphate-buffered saline. (D) Porosity of Collagen and Collagen-TCP matrices compared with $\beta$-TCP/Col and $\beta$-TCP/Col-TCP hybrid constructs. (E,F) optical image of 2D cultured DPCs. (G) Alizarin Red S staining of 2D cultured DPCs after three weeks osteogenic differentiation (scale bar is $400 \mu \mathrm{m}) .(H)$ Immunofluorescence images of 2D cultured DPCs using surface marker of CD90 and DAPI (scale bar is $400 \mu \mathrm{m}$ ). (I) Fluorescence activated cell sorting (FACS) of cell-surface markers in DPCs defined them to be positive for CD44, CD90 and CD105, while they are negative towards haematopoietic markers of CD34, CD45.

The porosity of Collagen and Collagen-TCP matrices were compared with $\beta$-TCP/Col and $\beta$-TCP/Col-TCP hybrid constructs in Fig. 4D. As displayed, the porosity of Collagen and Collagen-TCP matrices is reduced from $97.7 \pm 0.4 \%$ and $97.2 \pm 0.3 \%$ to $42.1 \pm 0.9 \%$ and $42.2 \pm 3.1 \%$ after incoporation into the 3D-printed $\beta$-TCP scaffold.

As shown in Fig. 4E, F, the isolated DPCs have formed fibroblast-like morphology. The osteogenic differentiation of DPCs was confirmed with the positive Alizarin Red S staining shown in Fig. 4G. DPCs were also investigated for their surface markers. Fig. $4 \mathrm{H}$ shows the immunofluorescence images of 2D cultured DPCs using positive surface marker CD90 and DAPI. Our obtained results from fluorescence activated cell sorting analysis of DPCs presented in Fig. 4I defined them to be positive for CD44, CD90 and CD105, while they are negative towards haematopoietic markers of CD34, CD45. The percentage of DPCs, positive for CD90, CD44 and CD105 was 99.7\%, $89.6 \%$ and $91.3 \%$ respectively.

Hematoxylin and eosin (H\&E) staining of cultured DPCs onto 3D-printed $\beta$-TCP/Col-TCP construct after three weeks is shown in Fig. $5 \mathrm{~A}$. As can be seen, the DPCs were attached and well proliferated onto the construct. This observation implies the interconnected porosity in constructs has allowed for the flux of nutrition inside the scaffold and removal of waste produced by the cells. As described above, the $\beta$-TCP/Col heterophasic constructs were designed to mimic the native bone ECM and endow a 3D microenvironment for DPCs. Fig. 5B-E shows the scanning electron micrographs for 3D-printed $\beta$-TCP scaffold, and 3D-printed $\beta$-TCP/Col-TCP construct seeded with DPCs after three weeks, respectively. As can be seen, the incorporation of the freeze-dried Col matrix into the $\beta$-TCP scaffold resulted in considerably improved attachment and proliferation of DPCs, which implies the enhanced seeding efficiency onto the hybrid constructs. 

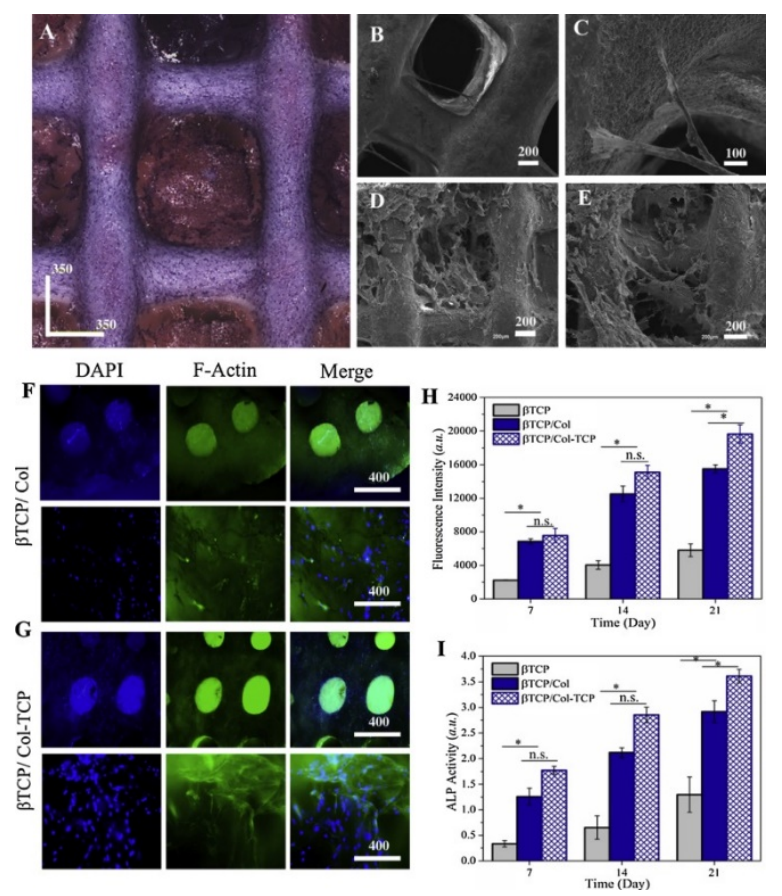

Fig. 5. (A) Hematoxylin and eosin (H\&E) staining of DPCs seeded onto 3D-printed $\beta$-TCP/Col construct after three weeks. Scanning electron micrographs of (B-C) 3D-printed $\beta$-TCP scaffold, and (D-E) 3D-printed $\beta$-TCP/Col-TCP construct seeded with DPCs after three weeks (B, C: scale bar is $500 \mu \mathrm{m} ; \mathrm{D}$, E: scale bar is $200 \mu \mathrm{m}$ ). The immunofluorescent images of encapsulated DPCs into (F) $\beta$-TCP/Col, and (G) $\beta$-TCP/Col-TCP constructs after 3 weeks. (H) DPCs viability cultured onto 3D-printed $\beta$-TCP scaffold in comparison with $\beta$-TCP/Col and $\beta$-TCP/ColTCP constructs after over three weeks. (I) Alkaline phosphatase (ALP) activity of DPCs cultured onto 3D-printed $\beta$-TCP scaffold in comparison with $\beta$-TCP/Col and $\beta$-TCP/Col-TCP constructs after 1,2 , and 3 weeks.

The immunofluorescent images of cultured DPCs onto $\beta$-TCP/Col and $\beta$-TCP/Col-TCP constructs after 3 weeks are displayed in Fig. 5F, G. As evident, the DPCs proliferated more onto the $\beta$-TCP/Col-TCP than $\beta$-TCP/Col construct after 3 weeks. In order to get a quantitative insight into cells proliferation, the viability of DPCs cultured onto the 3D-printed $\beta$-TCP scaffold was compared with $\beta$-TCP/Col and $\beta$-TCP/Col-TCP constructs over three weeks. Fig. $5 \mathrm{H}$ demonstrate significantly improved proliferation of DPCs cultured onto the $\beta-\mathrm{TCP} / \mathrm{Col}$ and $\beta$-TCP/Col-TCP hybrid constructs compared with the $\beta$-TCP scaffold. As can be seen in Fig. 5I, the increased ALP expression indicates that cells underwent differentiation and induced osteogenic markers. Similarly for 3Dprinted $\beta$-TCP scaffold, as well as $\beta$-TCP/Col and $\beta$-TCP/Col-TCP constructs a time dependant increase of DPCs differentiation was observed. The ALP activity of DPCs cultured onto the $\beta$-TCP/Col-TCP was found to be significantly higher than the other groups.

\section{Discussion}

The flowability of designed $\beta$-TCP-based ink formulations was optimized for the 3D-printing process. According to the formulations described in the method section, the $\beta$-TCP-based paste inks were composed of constant amount of $\beta$-TCP, carboxymethylcellulose (CMC) and sodium tripolyphospahte (TPP), but different volume of water. In the presented ink formulations CMC and TPP act as the binder and viscosity modifier, respectively; while various amounts of water were intended to tune the flow-ability of the paste and merging of strands during 3D-printing process. The shear sweep measurements revealed a yield stress and a shear-thinning behaviour for the paste formulations, which were found to be necessary for the 3D-printing process. The shear viscosity results at various temperatures from $17^{\circ} \mathrm{C}$ to $26^{\circ} \mathrm{C}$ indicated the reduction of shear viscosity with 
increasing temperature. This observation is closely related to the screening of hydrogen bonds among binder CMC chains, which facilitates the flow-ability of the paste ink.

The quality of the isolated Collagen was verified by the differential scanning calorimetry (DSC) method through assessment of its denaturation behaviour. Collagen chains undergo denaturation due to breaking of various intermolecular interactions including disulfide bonds, glycosylation of lysine and hydroxylysine residues [32], [33]. It has been shown that water residue plays a substantial role in thermal stability of Collagen fibres, in which dehydration considerably upsurges thermal denaturation of the Collagen fibrillar network [34], [35]. By increasing temperature, the intramolecular hydrogen bonds stabilizing Collagen chains are disappeared; hence, the triple helix structures collapse [36] [37].

Inspired by the native bone extracellular matrix, which is composed of the mineral phase embedded into a mainly collagenous network, TCP was incorporated into freeze-dried Collagen matrix in order to improve the osteogenic potential of the designed heterophasic constructs. The presented methodology offers combined advantages of the 3D porous ECM-like Collagen matrix for cell growth and proliferation, supported by 3D printed $\beta$-TCP scaffold. It is worth to note that the inclusion effect of $\beta$-TCP added into the Collagen matrix on the porosity was not significant. This indicates marginal influence of $\beta$-TCP on pore formation in Collagen matrix by freeze-drying method. The porosity of the $\beta-\mathrm{TCP} / \mathrm{Col}$ constructs resembles proximal tibial trabecular bone, which has typically $50-90 \%$ porosity.

As expected, a drastic difference in compressive modulus of freeze-dried Collagen-based matrices and the hybrid constructs was observed. It was found that the compressive modulus of freeze-dried Collagen-based matrices increases to more than 3 orders of magnitude after inclusion into the 3D-printed $\beta$-TCP scaffolds. In order to get an insight into the fate of 3D-printed $\beta$-TCP scaffolds responsible for the mechanical function of the hybrid constructs, their compressive modulus over 4 weeks immersion in PBS was compared with the dry state. The 3D-printed $\beta$-TCP scaffolds were found to preserve more than $30 \%$ of their initial compressive modulus, which allows for ingrowth of the cells into the constructs and gradual replacement of the regenerated bone tissue.

Isolation of human dental pulp cells (DPCs) as an odontogenic progenitor from impacted adult dental pulp tissue was first reported by Gronthos et al in 2000 [39]. Over the past years, DPCs have shown great capacity for bone regeneration owing to their potential to provide osteoinductive bone factors and differentiation

to osteoblasts in vitro and in vivo [40]. The isolated DPCs have formed fibroblast-like morphology, as reported by other researchers [39]. DPCs have both several similarities and differences to bone marrow stromal cells (BMSCs) [41]. DPCs have significantly higher colony-forming ability and number of proliferating cells compared to BMSCs over similar plating densities [39]. After three weeks osteogenic differentiation, Alizarin Red $S$ staining, as a functional assay, was performed on cultured DPCs to assess their differentiation capacity. Kawashima has discussed the typical surface markers of DPCs, and defined them to be positive for CD44, CD73, CD90 and CD105, while they are negative towards haematopoietic markers of CD34, CD45, and HLA-DR [42]. In consistent with these features, the presented results from the fluorescence activated cell sorting analysis of DPCs converge to indicate the successful DPCs isolation.

It was found that the hybrid constructs offer drastically improved cell viability compared to the pristine $\beta$-TCP scaffold. Such capability originates from the implemented combinatorial fabrication technique through inclusion of the freeze-dried Collagen matrix into the 3D-printed mineral support. Although no significant difference was observed between the $\beta$-TCP/Col and $\beta$-TCP/Col-TCP in the term of cell viability over two weeks, but $\beta$-TCP/ColTCP showed superior proliferation after three weeks post culture time. Such an improvement could be associated with the incorporation of $\beta$-TCP phase into the Collagen matrix. 
Alkaline phosphatase (ALP) is an early osteogenic differentiation marker and also an ectoenzyme elaborating degradation of inorganic pyrophosphate involved in release of phosphate for bone mineralization [43]. In order to assess the designed constructs as osteogenic platform for DPCS, their (ALP) activity as one of the early osteogenic differentiation markers was measured over three weeks. The increased ALP expression indicates that cells underwent differentiation and induced osteogenic markers. Similarly for 3D-printed $\beta$-TCP scaffold, as well as $\beta$-TCP/Col and $\beta$-TCP/Col-TCP constructs a time dependant increase of DPCs differentiation was observed. The ALP activity of DPCs cultured onto $\beta$-TCP/Col-TCP was found to be significantly higher than other groups. Such effect resembles the osteogenic differentiation of hMSCs promoted by the CaP-rich mineral microenvironment in bone ECM via phosphate-ATP-adenosine metabolic signaling [44]. Indeed, the dynamic dissolution/precipitation of $\mathrm{CaP}$ minerals governs the $\mathrm{Ca}^{2+}$ and $\mathrm{PO} 43$ - ions concentration in the $\mathrm{ECM}$. It has been shown that cells uptake the PO43-ions through the solute carrier family 20 (phosphate transporter), member 1 (SLC20a1) and then enters the mitochondria for ATP synthesis. The secreted ATP is subsequently metabolized into adenosine, which promotes osteogenic differentiation of hMSCs. These results are in accordance with other reports [45], [46].

\section{Conclusions}

A major challenge in scaffold design for bone tissue engineering is to employ various fabrication techniques to achieve platforms with combined mechanical function and regenerative capacity. In this study, a combinatorial fabrication approach based on the successive employment of 3D-printingtechnique and freeze-drying method was developed to design heterophasic constructs for osteoblastic differentiation of dental pulp stem cells (DPCs). Inspired by the anisotropic microstructure of the native bone tissues, the designed constructs were consisted of a 3D-printed $\beta$-TCP scaffold with interconnected porosity resembling the bone mineral phase to fulfill the mechanical function, embedded with a collagenous matrix to mimic the bone extracellular matrix and support DPCs adhesion, proliferation, and osteogenic differentiation. The increased expression of alkaline phosphatase (ALP) as an early osteogenic marker by DPCs seeded on $\beta$-TCP/Col-TCP scaffolds demonstrated their capability as promising platforms for bone tissue engineering. The obtained results demonstrated a great potential of the developed constructs for regeneration of craniomaxillofacial bone defects.

\section{Acknowledgment}

The authors would like to thank the financial supports from Marquette Innovation Fund, NIH/NICDRgrant DE018250, Osteo Science Foundation (Peter Geistlich Award), Delta Dental, AFOSR (ㄷ99550-12$\underline{1-0225}$ ) and NSF (EEC-1160483, ECCS-1351533 and CMMI-1363485).

\section{References}

[1] R. Tare, J. Kanczler, A. Aarvold, A. Jones, D. Dunlop, R. Oreffo, et al. Skeletal stem cells and bone regeneration: translational strategies from bench to clinic Proc Inst Mech Eng H: J Eng Med, 224 (2010), pp. $1455-1470$

[2] T. Kurien, R. Pearson, B. Scammell Bone graft substitutes currently available in orthopaedic practice Bone Joint J, 95 (2013), pp. 583-597

[3] S. Pina, J.M. Oliveira, R.L. Reis Natural-based nanocomposites for bone tissue engineering and regenerative medicine: a review Adv Mater, 27 (2015), pp. 1143-1169

[4] S. Bose, S. Tarafder, A. Bandyopadhyay Effect of chemistry on osteogenesis and angiogenesis towards bone tissue engineering using 3D printed scaffolds Ann Biomed Eng, 45 (2017), pp. 261-272

[5] M.M. Hasani-Sadrabadi, E. Dashtimoghadam, G. Bahlakeh, F.S. Majedi, H. Keshvari, J.J.Van Dersarl, et al. Onchip synthesis of fine-tuned bone-seeking hybrid nanoparticles Nanomedicine, 10 (2015), pp. 34313449 
[6] M.M. Hasani-Sadrabadi, S. Taranejoo, E. Dashtimoghadam, G. Bahlakeh, F.S. Majedi, J.J. VanDersarl, et al. Microfluidic manipulation of core/shell nanoparticles for oral delivery of chemotherapeutics: a new treatment approach for colorectal cancer Adv Mater, 28 (2016), pp. 4134-4141

[7] A. Khojasteh, F. Fahimipour, M.B. Eslaminejad, M. Jafarian, S. Jahangir, F. Bastami, et al. Development of PLGA-coated $\beta$-TCP scaffolds containing VEGF for bone tissue engineering Mater Sci Eng: C, 69 (2016), pp. 780-788

[8] M. Razavi, M. Fathi, O. Savabi, D. Vashaee, L. Tayebi In vivo assessments of bioabsorbable AZ91 magnesium implants coated with nanostructured fluoridated hydroxyapatite by MAO/EPD technique for biomedical applications Mater Sci Eng: C, 48 (2015), pp. 21-27

[9] V. Karageorgiou, D. Kaplan Porosity of 3D biomaterial scaffolds and osteogenesis Biomaterials, 26 (2005), pp. 5474-5491

[10] S.C. Marks, P.R. Odgren Structure and development of the skeleton Princ Bone Biol, 1 (2002), pp. 3-15

[11] B. Leukers, H. Gülkan, S.H. Irsen, S. Milz, C. Tille, M. Schieker, et al. Hydroxyapatite scaffolds for bone tissue engineering made by 3D printing J Mater Sci, 16 (2005), pp. 1121-1124

[12] M.M. Hasani-Sadrabadi, S.P. Hajrezaei, S.H. Emami, G. Bahlakeh, L. Daneshmandi, E.Dashtimoghadam, et al. Enhanced osteogenic differentiation of stem cells via microfluidics synthesized nanoparticles Nanomed: Nanotechnol Biol Med, 11 (2015), pp. 1809-1819

[13] A. Khojasteh, F. Fahimipour, M. Jafarian, D. Sharifi, S. Jahangir, F. Khayyatan, et al. Bone engineering in dog mandible: coculturing mesenchymal stem cells with endothelial progenitor cells in a composite scaffold containing vascular endothelial growth factor J Biomed Mater Res B Appl Biomater, 105.7 (2017), pp. 1767-1777

[14] S.-S. Kim, M.S. Park, O. Jeon, C.Y. Choi, B.-S. Kim Poly(lactide-co-glycolide)/hydroxyapatite composite scaffolds for bone tissue engineering Biomaterials, 27 (2006), pp. 1399-1409

[15] F. Fahimipour, T.J. Kashi, K. Khoshroo, E. Dashtimoghadam, M. Rasoulianborujeji, L.Tayebi 3D-printed $\boldsymbol{\beta}$ TCP/collagen scaffolds for bone tissue engineering Dent Mater, 32 (2016), p. e57

[16] M. Yazdimamaghani, D. Vashaee, S. Assefa, M. Shabrangharehdasht, A.T. Rad, M.A.Eastman, et al. Green synthesis of a new gelatin-based antimicrobial scaffold for tissue engineering Mater Sci Eng: C, 39 (2014), pp. 235-244

[17] M. Razavi, M.H. Fathi, O. Savabi, D. Vashaee, L. Tayebi Biodegradation, bioactivity and in vivo biocompatibility analysis of plasma electrolytic oxidized (PEO) biodegradable Mg implants Phys Sci Int J, 4 (2014), p. 708

[18] H.E. Jazayeri, M. Tahriri, M. Razavi, K. Khoshroo, F. Fahimipour, E. Dashtimoghadam,et al. A current overview of materials and strategies for potential use in maxillofacial tissue regeneration Mater Sci Eng: C, 70 (2017), pp. 913-929

[19] T. Almela, I.M. Brook, K. Khoshroo, M. Rasoulianboroujeni, F. Fahimipour, M. Tahriri,et al. Simulation of cortico-cancellous bone structure by 3D printing of bilayer calcium phosphate-based scaffolds Bioprinting, 6 (2017), pp. 1-7

[20] K. Khoshroo, T. Almela, M. Tahriri, F. Fahimipour, Z. Metalwala, K. Moharamzadeh, et al. 3D-printing of porous calcium phosphate cements for bone tissue engineering Dent Mater, 32 (2016), pp. e56-e57

[21] S.C. Cox, J.A. Thornby, G.J. Gibbons, M.A. Williams, K.K. Mallick 3D printing of porous hydroxyapatite scaffolds intended for use in bone tissue engineering applications Mater Sci Eng: C, 47 (2015), pp. 237247

[22] E. Dashtimoghadam, F. Fahimipour, B. Davaji, M. Hasani-Sadrabadi, L. Tayebi Microfluidic-directed synthesis of polymeric nanoparticles for bone cancer therapy Dent Mater, 32 (2016), pp. e59-e60

[23] M.M. Aghdasi, M. Asnaashari, A. Aliari, F. Fahimipour, S. Soheilifar Conventional versus digital radiographs accuracy in detecting artificial voids in root canal filling material Iran Endod J, 6 (2011), pp. 99-102 
[24] S. Bose, S. Vahabzadeh, A. Bandyopadhyay Bone tissue engineering using 3D printing Mater Today, 16 (2013), pp. 496-504

[25] J.K. Sherwood, S.L. Riley, R. Palazzolo, S.C. Brown, D.C. Monkhouse, M. Coates, et al. A three-dimensional osteochondral composite scaffold for articular cartilage repair Biomaterials, 23 (2002), pp. 4739-4751

[26] B.C. Gross, J.L. Erkal, S.Y. Lockwood, C. Chen, D.M. Spence Evaluation of 3D printing and its potential impact on biotechnology and the chemical sciences ACS Publications (2014)

[27] N. Rajan, J. Habermehl, M.-F. Coté, C.J. Doillon, D. Mantovani Preparation of ready-to-use, storable and reconstituted type I collagen from rat tail tendon for tissue engineering applications Nat Protoc, 1 (2006), p. 2753

[28] A. Shahini, M. Yazdimamaghani, K. Walker, M. Eastman, H. Hatami-Marbini, B. Smith,et al. 3D conductive nanocomposite scaffold for bone tissue engineering Int J Nanomed, 9 (2014), pp. 167-181

[29] W. Xue, A. Bandyopadhyay, S. Bose Polycaprolactone coated porous tricalcium phosphate scaffolds for controlled release of protein for tissue engineering J Biomed Mater Res B Appl Biomater, 91 (2009), pp. 831-838

[30] K. Payne, A. Veis Fourier transform IR spectroscopy of collagen and gelatin solutions: deconvolution of the amide I band for conformational studies Biopolymers, 27 (1988), pp. 1749-1760

[31] A.P.D. Moreira, M.S. Sader, G.D.d.A. Soares, M.H.M.R. Leão Strontium incorporation on microspheres of alginate/ $\beta$-tricalcium phosphate as delivery matrices Mater Res, 17 (2014), pp. 967-973

[32] C.A. Miles, A.J. Bailey Thermal denaturation of collagen revisited J Chem Sci, 111 (1999), pp. 71-80

[33] S.P. Robins, A.J. Bailey The chemistry of the collagen cross-links: the mechanism of stabilization of the reducible intermediate cross-links Biochem J, 149 (1975), pp. 381-385

[34] C. Miles, T. Burjanadze Thermal stability of collagen fibers in ethylene glycol Biophys J, 80 (2001), pp. 14801486

[35] C.A. Miles, N.C. Avery, V.V. Rodin, A.J. Bailey The increase in denaturation temperature following crosslinking of collagen is caused by dehydration of the fibres J Mol Biol, 346 (2005), pp. 551-556

[36] R. Fraser, T. MacRae, E. Suzuki Chain conformation in the collagen molecule J Mol Biol, 129 (1979), pp. 463481

[37] C.A. Miles, T.V. Burjanadze, A.J. Bailey The kinetics of the thermal denaturation of collagen in unrestrained rat tail tendon determined by differential scanning calorimetry J Mol Biol, 245 (1995), pp. 437-446

[39] S. Gronthos, M. Mankani, J. Brahim, P.G. Robey, S. Shi Postnatal human dental pulp stem cells (DPSCs) in vitro and in vivo Proc Natl Acad Sci, 97 (2000), pp. 13625-13630

[40] J.M. Seong, B.-C. Kim, J.-H. Park, I.K. Kwon, A. Mantalaris, Y.-S. Hwang Stem cells in bone tissue engineering Biomed Mater, 5 (2010), p. 062001

[41] M. Tatullo, M. Marrelli, K.M. Shakesheff, L.J. White Dental pulp stem cells: function, isolation and applications in regenerative medicine J Tissue Eng Regen Med, 9 (2015), pp. 1205-1216

[42] N. Kawashima Characterisation of dental pulp stem cells: a new horizon for tissue regeneration Arch Oral Biol, 57 (2012), pp. 1439-1458

[43] U. Stucki, J. Schmid, C.F. Hämmerle, N.P. Lang Temporal and local appearance of alkaline phosphatase activity in early stages of guided bone regeneration Clin Oral Implants Res, 12 (2001), pp. 121-127

[44] Y.-R.V. Shih, Y. Hwang, A. Phadke, H. Kang, N.S. Hwang, E.J. Caro, et al .Calcium phosphate-bearing matrices induce osteogenic differentiation of stem cells through adenosine signaling Proc Natl Acad Sci, 111 (2014), pp. 990-995

[45] S.A. Ajlan, N.Y. Ashri, A.M. Aldahmash, M.S. Alnbaheen Osteogenic differentiation of dental pulp stem cells under the influence of three different materials BMC Oral Health, 15 (2015), p. 132

[46] R. Khanna-Jain, B. Mannerström, A. Vuorinen, G.K. Sándor, R. Suuronen, S. Miettinen Osteogenic differentiation of human dental pulp stem cells on $\beta$-tricalcium phosphate/poly(I-lactic acid/caprolactone) three-dimensional scaffolds J Tissue Eng, 3 (2012) 2041731412467998 
